\title{
Determination of the Spin-Rotation Fine Structure of $\mathrm{He} 2+$
}

\section{Journal Article}

\section{Author(s):}

Jansen, Paul; Semeria, Luca; Merkt, Frédéric

Publication date:

2018-01-26

Permanent link:

https://doi.org/10.3929/ethz-b-000238549

\section{Rights / license:}

In Copyright - Non-Commercial Use Permitted

\section{Originally published in:}

Physical Review Letters 120(4), https://doi.org/10.1103/PhysRevLett.120.043001

\section{Funding acknowledgement:}

172620 - Precision measurements with cold molecules: Rydberg states, ions and photoionization (SNF) 743121 - Cold Ion Chemistry - Experiments within a Rydberg Orbit (EC) 
This article may be downloaded for personal use only. Any other use requires prior permission of the author and The American Physical Society (APS).

The following article appeared in Phys. Rev. Lett. 120, 043001 (2018) and may be found at https://journals.aps.org/prl/abstract/10.1103/PhysRevLett.120.043001. 


\title{
Determination of the spin-rotation fine structure of $\mathrm{He}_{2}{ }^{+}$
}

\author{
Paul Jansen, Luca Semeria, and Frédéric Merkt* \\ Laboratory of Physical Chemistry, ETH Zurich, CH-8093 Zurich, Switzerland
}

(Dated: December 18, 2017)

\begin{abstract}
Measuring spin-rotation intervals in molecular cations is challenging, particularly so when the ions do not have electric-dipole-allowed rovibrational transitions. We present a method, based on an angular-momentum basis transformation, to determine the spin-rotational fine structure of molecular ions from the fine structure of high Rydberg states. The method is illustrated by the determination of the so far unknown spin-rotation fine structure of the fundamentally important $\mathrm{He}_{2}{ }^{+}$ion in the $\mathrm{X}^{2} \Sigma_{\mathrm{u}}^{+}$state. The fine-structure splittings of the $v^{+}=0, N^{+}=1,3$, and 5 levels of $\mathrm{He}_{2}{ }^{+}$are $7.96(14) \mathrm{MHz}, 17.91(32) \mathrm{MHz}$ and $28.0(6) \mathrm{MHz}$, respectively. The experiment relies on the use of single-mode cw radiation to record spectra of high Rydberg states of $\mathrm{He}_{2}$ from the a ${ }^{3} \Sigma_{\mathrm{u}}^{+}$ metastable state.
\end{abstract}

$\mathrm{He}_{2}{ }^{+}$is a structurally simple molecular system and was one of the first molecules to be studied by $a b$ initio quantum-mechanical methods $[1,2]$. Its $\mathrm{X}{ }^{2} \Sigma_{\mathrm{u}}^{+}$ electronic ground state has the electronic configuration $\left(1 \sigma_{\mathrm{g}}\right)^{2}\left(1 \sigma_{\mathrm{u}}\right)^{1}$, with two electrons in the $1 \sigma_{\mathrm{g}}$ bonding orbital and one electron in the antibonding $1 \sigma_{\mathrm{u}}$ orbital. Its dissociation energy $D_{0}\left({ }^{4} \mathrm{He}_{2}^{+}\right) / h c$ and equilibrium internuclear separation $R_{\mathrm{e}}$ are $19116.116 \mathrm{~cm}^{-1}$ and $2.042 a_{0}$, respectively $[3,4] . \mathrm{He}_{2}{ }^{+}$is encountered in He-containing plasmas [5], is a central structural element in positively charged helium clusters [6], and is thought to have been one of the first molecules formed in the early universe [7].

The small charge of the He nuclei and the small number of electrons make $\mathrm{He}_{2}{ }^{+}$one of the few molecules, next to $\mathrm{H}_{2}{ }^{+}, \mathrm{H}_{2}, \mathrm{HHe}^{+}, \mathrm{LiH}^{+}$, and $\mathrm{LiH}$ for which exact quantum-mechanical computations are in principle possible (see, e.g., Refs. 4, 8-10). The comparison of the results of such computations with the results of spectroscopic measurements offers the prospects of either identifying incomplete aspects of the theoretical treatment or uncovering new effects. The rapidly growing efforts invested in such comparisons are part of the search for what is referred to as "physics beyond the standard model" using molecules [11, 12]. Such searches exploit molecular systems to attempt, e.g., the observation of so far unknown forces [13] or of compactified higher dimensions [14]. Precision spectroscopy in simple few-electron molecules may also contribute to resolve what is known as the proton-charge-radius puzzle $[15,16]$. A crucial aspect of these efforts is the establishment and testing of reliable and highly accurate computational procedures, which is best done with the simplest molecules.

Until recently, the experimental data available on the energy-level structure of $\mathrm{He}_{2}{ }^{+}$consisted of only 16 transitions [17-19]: 9 rovibrational transitions in the IR connecting rotational levels of the ground $(v=0)$ and first excited $(v=1)$ vibrational level of ${ }^{3} \mathrm{He}^{4} \mathrm{He}^{+}[17,18]$, which, unlike ${ }^{4} \mathrm{He}_{2}{ }^{+}$, possesses a permanent electric dipole moment, and 7 electronic transitions in the microwave range connecting weakly bound rovibrational levels of the $\mathrm{X}^{2} \Sigma_{\mathrm{u}}^{+}$and $\mathrm{A}^{2} \Sigma_{\mathrm{g}}^{+}$electronic states of ${ }^{4} \mathrm{He}_{2}^{+}$
[19]. These data could be fully accounted for by the latest $a b$ initio calculations [4]. They have since been extended by precision measurements of the positions of many rotational levels of the ground vibronic state of ${ }^{4} \mathrm{He}_{2}{ }^{+}$, which revealed systematic and increasing deviations between calculated and experimental energies with increasing rotational excitation [20-23].

No information has been obtained so far on the spinrotation splittings of the $\mathrm{He}_{2}{ }^{+}$rotational levels. $\mathrm{Yu}$ et al. [18] could measure the hyperfine structure in the IR spectrum of ${ }^{3} \mathrm{He}^{4} \mathrm{He}^{+}$arising from the $I=1 / 2$ nuclear spin of ${ }^{3} \mathrm{He}$, but did not resolve the spin-rotation splittings. A calculation of the spin-rotation interaction indicated a spin-rotation coupling constant $\gamma^{+}\left({ }^{3} \mathrm{He}^{4} \mathrm{He}^{+}\right)$of about $-3.5 \mathrm{MHz}[18]$, too small to be observed in the IR spectra. Taking the $\mu^{-1}$ dependence of the spin-rotation coupling constant on the reduced mass $\mu$, a value of about $-3 \mathrm{MHz}$ can be predicted for $\gamma^{+}\left({ }^{4} \mathrm{He}_{2}{ }^{+}\right)$, more than 10 times less than in the ground state of $\mathrm{H}_{2}^{+}$[24-26]. The contribution to the spin-rotation constant from the secondorder spin-orbit coupling $[27,28]$ is small in $\mathrm{He}_{2}{ }^{+}$because of the large energetic separation of the ground state from excited electronic states. From a precision measurement of the spin-rotation intervals, one may determine the frequencies of the radiowave magnetic transitions between the spin-rotation components, through which $\mathrm{He}_{2}{ }^{+}$ might be detected.

We demonstrate here a method to determine the spinrotation splittings of the rotational levels of molecular cations from the fine structure of high Rydberg states and illustrate it by a measurement of the spin-rotation splittings of $\mathrm{He}_{2}{ }^{+}$. Their small size necessitates the use of narrow-band cw laser radiation to record the Rydberg spectrum at high values of the principal quantum number, which, to our knowledge, has not yet been possible in any molecular system.

We recorded the Rydberg spectum of $\mathrm{He}_{2}$ from the metastable a ${ }^{3} \Sigma_{\mathrm{u}}^{+}$state $\left(\mathrm{He}_{2}^{*}\right.$ hereafter) in a supersonic beam [29] using the spectrometer described in Ref. 30. The single-mode cw UV laser radiation (bandwidth $2 \mathrm{MHz}$, power $300 \mathrm{~mW}$ ) used to record the spectrum 


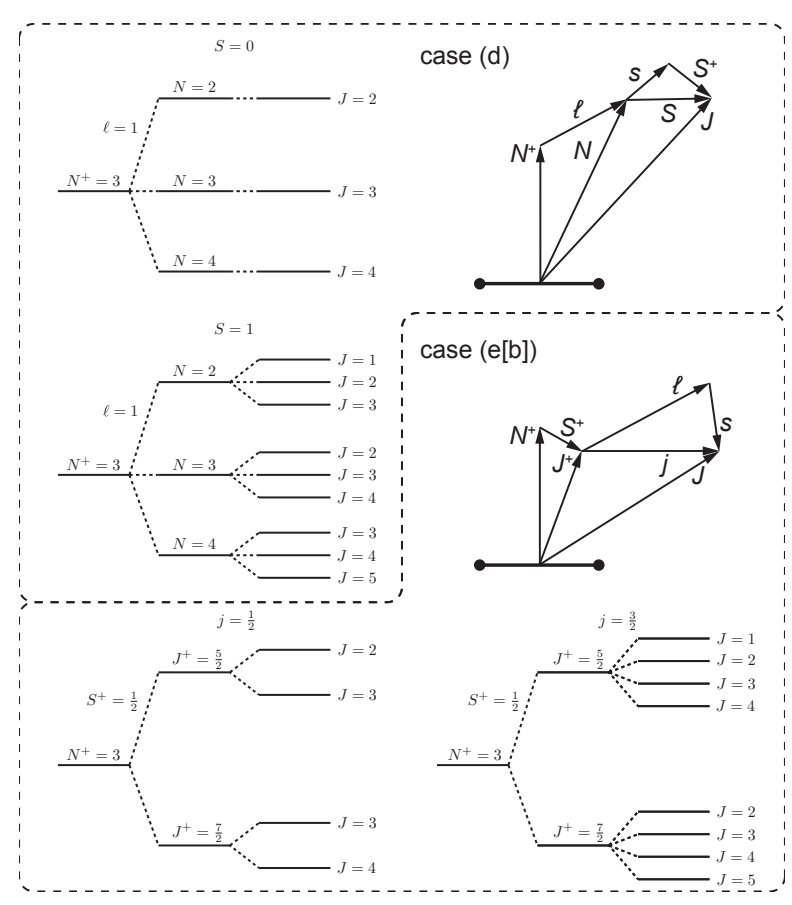

Figure 1. Hund's coupling cases (d) and (e[b]) and corresponding level structure (not to scale) of high Rydberg states of $\mathrm{He}_{2}$.

was generated by frequency doubling the output of a ring dye laser in a nonlinear crystal placed in an enhancement cavity. The perpendicular arrangement of the $\mathrm{He}_{2}^{*}$ and UV-laser beams reduced the Doppler width to $25 \mathrm{MHz}$ and the precision of the fine-structure intervals to about $2.5 \mathrm{MHz}$ [30]. Transitions were detected by delayed pulsed-field ionization of the Rydberg states. Spectra of selected transitions are depicted in Fig. 2. Most of the measurements were carried out at $n$ values around 50 except for weak series, for which measurements had to be performed at $n \approx 30$, and for strong series, for which measurements could also be performed at $n$ values up to 100.

Because the total molecular wavefunction must be symmetric under exchange of the spinless bosonic ${ }^{4} \mathrm{He}^{2+}$ nuclei, only odd rotational levels exist in $\mathrm{He}_{2}^{*}$ and in the $\mathrm{X}^{+2} \Sigma_{\mathrm{u}}^{+}$electronic ground state of $\mathrm{He}_{2}{ }^{+}$. In the following, double-prime symbols, unprimed symbols, and a superscript "+" are used to designate the quantum numbers of $\mathrm{He}_{2}^{*}, \mathrm{He}_{2}$ Rydberg states, and $\mathrm{He}_{2}{ }^{+}$, respectively. The level structure of $\mathrm{He}_{2}^{*}$ is best described using Hund's angular-momentum coupling case (b), where the total angular momentum excluding spin $\vec{N}^{\prime \prime}$ couples to the total electron spin $\vec{S}^{\prime \prime}$ to form the total angular momentum $\vec{J}^{\prime \prime}$. The spin-spin and spin-rotation interaction split each rotational level into three components with $J^{\prime \prime}=N^{\prime \prime}, N^{\prime \prime} \pm 1$. The characteristic spacing of the resulting fine structure of $\mathrm{He}_{2}^{*}$ has been measured for several rovibrational levels [31-35] and is dominated by the spin-spin interaction, which is two orders of magnitude stronger than the spin-rotation interaction [35]. The level structure of the $\mathrm{X}^{+2} \Sigma_{\mathrm{u}}^{+}\left(v^{+}=0\right)$ state of $\mathrm{He}_{2}{ }^{+}$is also best described using Hund's case (b). The rotational levels are split into two fine-structure components by

$\tilde{\nu}_{\mathrm{sr}}^{+}=\gamma_{N^{+}}^{+}\left(N^{+}+\frac{1}{2}\right)$, with $\gamma_{N^{+}}^{+}=\gamma^{+}+\gamma_{\mathrm{D}}^{+} N^{+}\left(N^{+}+1\right)$.

In the absence of $\ell$ mixing, The angular-momentum coupling scheme of the Rydberg states of $\mathrm{He}_{2}$ depends on the relative strength of five competing interactions: (i) the exchange interaction between Rydberg and core electrons, (ii) the spin-rotation interaction of the ion core, (iii) the spin-spin interaction, (iv) the spin-orbit interaction, and (v) the $\ell$-uncoupling interaction that is responsible for the transition from Hund's case (b) to case (d) $[36,37]$ and is complete for $n \gtrsim 10$. Because of the small nuclear charge of $\mathrm{He}$, the spin-orbit interaction is completely negligible. The spin-spin interaction scales as $n^{-3}$ and is negligible in Rydberg states with $n>10$. The interactions that are responsible for the level structure in the Rydberg states of $\mathrm{He}_{2}$ are the spin-rotation interaction of the ion core, which is $n$ independent, and the exchange interaction, which splits the $n$ p Rydberg states in a singlet and a triplet state by

$$
\Delta E_{\mathrm{ex}} \approx \Delta \delta^{(\mathrm{S}, \mathrm{T})} \frac{2 h c \mathcal{R}_{\mathrm{He}_{2}}}{n^{3}}
$$

where $\Delta \delta^{(\mathrm{S}, \mathrm{T})}$ is the difference between the Hund's case (d) quantum defects, $\delta_{N, S}$, of the singlet and triplet states, and $\mathcal{R}_{\mathrm{He}_{2}}$ is the mass-corrected Rydberg constant for $\mathrm{He}_{2}$. Taking $\gamma^{+}$from $\mathrm{Yu}$ et al. [18] and estimating $\Delta \delta^{(\mathrm{S}, \mathrm{T})}$ from the $a b$ initio potential curves of Yarkony [38], Eqs. (1) and (2) predict that the spin-rotation interaction in the core is much less than the exchange interaction in Rydberg states with $n<150$, so that the total electron spin $S$ is a good quantum number in this range. The Rydberg states are adequately described in Hund's case (d), where $\vec{N}^{+}$couples to $\vec{\ell}$ to form $\vec{N}$, which couples to $\vec{S}$ to give $\vec{J}$ [see Fig. 1a)]. Above about $n=150$, $\vec{N}^{+}$couples to the spin of the core $\vec{S}^{+}$, which mixes singlet and triplet states [39], to form $\vec{J}^{+}, \vec{\ell}$ couples to the spin of the Rydberg electron $\vec{s}$ and the resulting vector $\vec{j}$ couples to $\vec{J}^{+}$to form $\vec{J}$. This situation corresponds to Hund's case (e[b]), depicted in Fig. 1b). When $n$ approaches infinity, the different $J$ levels originating from the same $J^{+}$value are split by the spin-rotation intervals of the ion levels. The Rydberg transitions observed in our spectra connect the Hund's case (b) levels of $\mathrm{He}_{2}^{*}$ to the Hund's case (d) Rydberg levels and can be labelled as $n \mathrm{p} N_{N, J}^{+} \leftarrow N_{J^{\prime \prime}}^{\prime \prime}$.

Hund's case (d), $\left|N^{+} N S\right\rangle=|N S\rangle$, and Hund's case (e[b]), $\left|N^{+} J^{+} j\right\rangle=\left|J^{+} j\right\rangle$, basis functions, as defined in Ref. [40], are related by the angular-momentum basis 

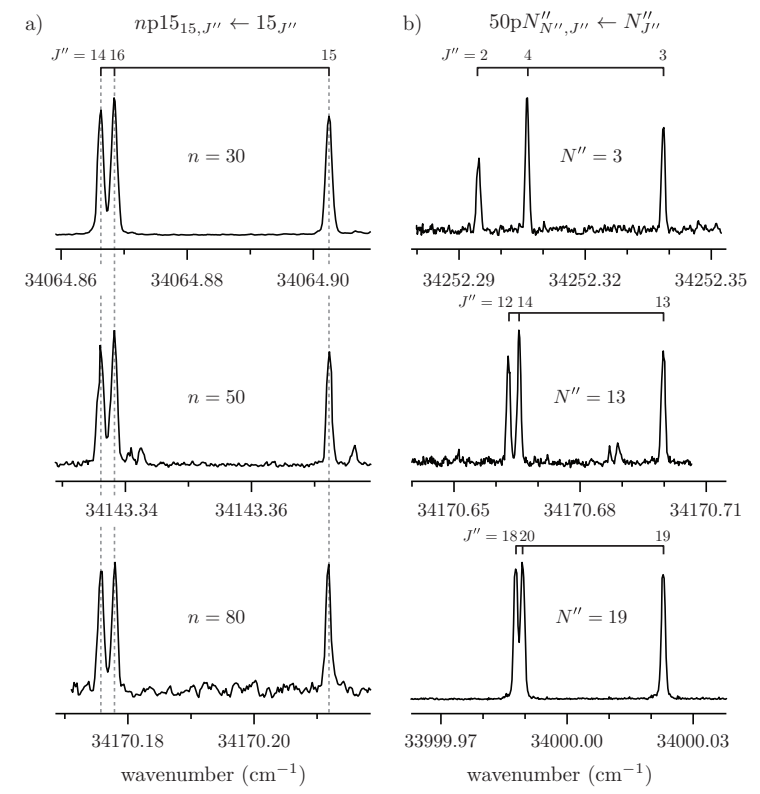

Figure 2. a) Rydberg spectra of $n \mathrm{p} 15_{15} \leftarrow 15$ transitions with $n=30,50$, and 80. b) Spectra of $50 \mathrm{p} N_{N^{\prime \prime}}^{\prime \prime} \leftarrow N^{\prime \prime}$ with $N^{\prime \prime}=3,13$, and 19. See text for details.

transformation

$$
\begin{gathered}
\mathcal{U} \equiv\left\langle J^{+} j \mid N S\right\rangle^{\left(J M_{J} p \Lambda^{+} N^{+} S^{+} q^{+} \ell s\right)}= \\
\sqrt{(2 j+1)\left(2 J^{+}+1\right)(2 S+1)(2 N+1)} \times \\
\left(\frac{1+\delta_{\Lambda^{+} 0}(-1)^{p-q^{+}-N^{+}+\ell}}{1+\delta_{\Lambda^{+}}}\right)\left\{\begin{array}{ccc}
N^{+} & S^{+} & J^{+} \\
\ell & s & j \\
N & S & J
\end{array}\right\},
\end{gathered}
$$

where $q^{+}=0$ and $p=1$ are the electronic core and total parity indices, respectively, $\Lambda^{+}=0$, and the last term is a Wigner-9j symbol. Equation (3) can be derived from the diagrams presented in Fig. 1 using standard angular-momentum algebra (see, e.g., Ref. 41). The fine structure of the $n \mathrm{p}$ Rydberg states of $\mathrm{He}_{2}$ for each set of $n$ and $N^{+}$values is derived from the eigenvalues of the $12 \times 12$ matrix

$$
\hat{\mathcal{H}}=\hat{\mathcal{H}}_{n, N^{+}, N, S}+\mathcal{U}^{\dagger} \hat{\mathcal{H}}_{\mathrm{sr}}^{+} \mathcal{U}
$$

where $\hat{\mathcal{H}}_{n, N^{+}, N, S}$ is a diagonal matrix describing the Hund's case (d) limit, with three sets of four elements for each value of $N\left(N=N^{+}, N^{+} \pm 1\right)$ : three degenerate elements corresponding to the triplet manifold $(S=1)$ and one to the singlet manifold $(S=0)$. Neglecting channel interactions, the diagonal elements are given by

$$
E_{n, N^{+}, N, S}=E_{N^{+}}-\frac{h c \mathcal{R}_{\mathrm{He}_{2}}}{\left(n-\delta_{N, S}\right)^{2}} .
$$

$\hat{\mathcal{H}}_{\mathrm{sr}}^{+}$is expressed in the Hund's case $(\mathrm{e}[\mathrm{b}])$ limit as a diagonal matrix with two sets of six degenerate elements, $\frac{1}{2} h c \gamma_{N^{+}}^{+} N^{+}$and $-\frac{1}{2} h c \gamma_{N^{+}}^{+}\left(N^{+}+1\right)$, corresponding to the
Table I. Spin-rotation intervals of high $n$ p Rydberg states as a function the spin-rotation coupling constant $\gamma^{+}$of the $\mathrm{X}$ ${ }^{2} \Sigma_{\mathrm{u}}^{+}$ion core.

\begin{tabular}{lccc}
\hline \hline & \multicolumn{3}{c}{$J$} \\
\cline { 2 - 4 }$N$ & $N-1$ & $N$ & $N+1$ \\
\hline$N^{+}-1$ & 0 & $\gamma^{+} \frac{N(N+2)}{2(N+1)}$ & $\gamma^{+\frac{(2 N+1)(N+2)}{2(N+1)}}$ \\
$N^{+}$ & 0 & $\gamma^{+\frac{N^{2}+N-1}{2(N+1)}}$ & $\gamma^{+\frac{\left(N^{2}+N-1\right)(2 N+1)}{2 N(N+1)}}$ \\
$N^{+}+1$ & 0 & $\gamma^{+\frac{N-1}{2}}$ & $\gamma^{+\frac{(2 N+1)(N-1)}{2 N}}$ \\
\hline \hline
\end{tabular}

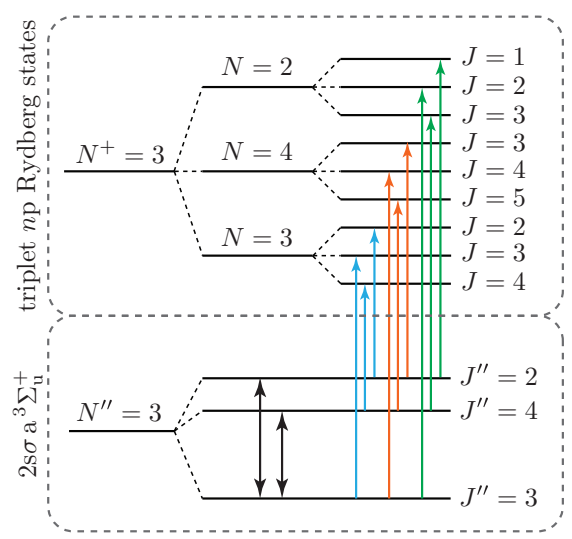

Figure 3. Illustration of the $\Delta J=\Delta N$ selection rules for Rydberg transitions from $N^{\prime \prime}=3$. The double-headed arrows indicate the fine-structure splittings in $\mathrm{He}_{2}^{*}$.

spin-rotation fine structure of the ion. A similar procedure has been used previously in the context of the case (b) to case (d) frame transformation and the rotational structure of $\mathrm{NO}^{+}[42]$ and $\mathrm{CaF}^{+}[43]$. Because the splittings of $n<150$ Rydberg states of different $N$ and $S$ values are much larger than the spin-rotation splitting of the ion, except near perturbations arising from channel interactions, the eigenvalues of Eq. (4) do not depend on $n$ nor on the quantum defects $\delta_{N, S}$. The relation between the fine-structure interval in the Rydberg states and the ion is thus purely geometrical and given by Eqs. (1), (3) and (4). This is illustrated in Fig. 2a), which compares the fine structure of the spectra of the $n \mathrm{p} 15_{15} \leftarrow 15$ for $n=30,50$, and 80 . Figure $2 \mathrm{~b}$ ) shows how the fine structure evolves with the value of $N^{\prime \prime}$. Analytic expressions for the fine-structure splittings of the Rydberg states resulting from Eqs. (1)-(5) are given in Table I. Compared to an extrapolation of the ionic fine structure by multichannel quantum-defect theory (MQDT), Eqs. (3) and (4) offer the advantage that they do not require knowledge of the quantum defects.

Transitions from $\mathrm{He}_{2}^{*}$ to $n \mathrm{p}$ Rydberg states are governed by selection rules. The transition electric dipole moment does not act on the spins, so that in this case $\Delta J=J-J^{\prime \prime}=N-N^{\prime \prime}=\Delta N$. The allowed transitions are depicted in Fig. 3 for $N^{\prime \prime}=3$. Their transition frequencies depend on the fine structures of both the metastable state and the Rydberg state. Because 
Table II. Experimental fine-structure intervals of triplet $n \mathrm{p}$ Rydberg states of $\mathrm{He}_{2}\left(\nu_{\mathrm{obs}}\right)$ and their comparison to the results of our model calculations $\left(\Delta=\nu_{\text {obs. }}-\nu_{\text {calc. }}\right)$. All values are given in $\mathrm{MHz}$.

\begin{tabular}{|c|c|c|c|c|}
\hline \multirow[b]{2}{*}{$n \mathrm{p} N_{N}^{+}$} & \multicolumn{2}{|c|}{$J=N \leftarrow J=N+1$} & \multicolumn{2}{|c|}{$J=N \leftarrow J=N-1$} \\
\hline & $\nu_{\mathrm{obs}}$ & $\Delta$ & $\nu_{\mathrm{obs}}$ & $\Delta$ \\
\hline $27 \mathrm{p} 1_{1}$ & $2(8)$ & 1.3 & $3(3)$ & 1.5 \\
\hline $49 \mathrm{p} 1_{1}$ & $4(3)$ & -1.1 & $-1(3)$ & -2.1 \\
\hline $50 \mathrm{p} 3_{3}$ & $5(3)$ & -4.7 & $7(3)$ & -0.4 \\
\hline $41 \mathrm{p} 3_{4}$ & $10(3)$ & 3.8 & $11(3)$ & 3.7 \\
\hline $98 \mathrm{p} 3_{4}$ & $8(4)$ & 3.6 & $13(5)$ & 4.9 \\
\hline $99 \mathrm{p} 3_{4}$ & $14(3)$ & 1.8 & $5(3)$ & -2.9 \\
\hline $101 \mathrm{p} 34$ & $16(4)$ & 9.9 & $11(4)$ & 3.1 \\
\hline $50 \mathrm{p} 5_{5}$ & $11(3)$ & -2.7 & $13(3)$ & 0.6 \\
\hline $44 \mathrm{p} 56$ & $11(3)$ & 3.4 & $20(3)$ & 7.3 \\
\hline $50 \mathrm{p} 7_{7}$ & $17(3)$ & 0.9 & $22(3)$ & 4.2 \\
\hline $50 \mathrm{p} 7_{8}$ & $24(3)$ & -0.1 & $14(3)$ & -3.9 \\
\hline $32 \mathrm{p} 99$ & $23(3)$ & 1.6 & $25(3)$ & 3.1 \\
\hline $50 \mathrm{p} 99$ & $23(3)$ & 1.6 & $25.7(15)$ & 3.4 \\
\hline $63 \mathrm{p} 99$ & $26(3)$ & 1.3 & $22.4(19)$ & 0.1 \\
\hline $63 \mathrm{p} 9_{10}$ & $25(4)$ & 3.1 & $26(4)$ & 3.2 \\
\hline $50 \mathrm{p} 11_{11}$ & $29(3)$ & -0.7 & $27(3)$ & -0.3 \\
\hline $35 \mathrm{p} 11_{12}$ & $32(3)$ & -1.0 & $24(3)$ & -3.5 \\
\hline $50 \mathrm{p} 13_{13}$ & $34(3)$ & 0.2 & $32(3)$ & 0.1 \\
\hline $54 \mathrm{p} 13_{13}$ & $27.6(25)$ & -2.5 & $36(3)$ & 4.0 \\
\hline $54 \mathrm{p} 13_{14}$ & $41(3)$ & 1.2 & $26(3)$ & -6.1 \\
\hline $30 \mathrm{p} 15_{15}$ & $36(3)$ & -0.7 & $38(3)$ & 1.7 \\
\hline $50 \mathrm{p} 15_{15}$ & $37(3)$ & -1.6 & $36(3)$ & 0.2 \\
\hline $54 \mathrm{p} 15_{15}$ & $37(3)$ & -3.1 & $35(3)$ & -1.3 \\
\hline $54 \mathrm{p} 15_{16}$ & $32.7(25)$ & -1.5 & $40.4(25)$ & 4.2 \\
\hline $55 \mathrm{p} 15_{15}$ & $34(4)$ & -0.7 & $40(5)$ & 4.0 \\
\hline $55 \mathrm{p} 15_{16}$ & $42(3)$ & 1.5 & $31(4)$ & -4.8 \\
\hline $80 \mathrm{p} 15_{15}$ & $32(3)$ & 0.8 & $43(3)$ & 6.7 \\
\hline $80 \mathrm{p} 15_{16}$ & $35.7(17)$ & -0.6 & $38(3)$ & 2.1 \\
\hline $30 \mathrm{p} 17_{17}$ & $41(3)$ & -1.6 & $40.3(25)$ & 0.1 \\
\hline $91 \mathrm{p} 17_{17}$ & $37(11)$ & -1.6 & $44(11)$ & 4.0 \\
\hline $91 \mathrm{p} 17_{18}$ & $41(3)$ & -0.2 & $41(3)$ & 0.9 \\
\hline $30 \mathrm{p} 19_{19}$ & $47(3)$ & -2.8 & $41(3)$ & -3.4 \\
\hline $30 \mathrm{p} 21_{21}$ & $51(3)$ & -2.2 & $44(3)$ & -3.5 \\
\hline $30 \mathrm{p} 23_{23}$ & $53(3)$ & -2.7 & $48(3)$ & -2.5 \\
\hline $30 \mathrm{p} 25_{25}$ & $55(3)$ & 0.3 & $55(3)$ & 1.3 \\
\hline $30 \mathrm{p} 27_{27}$ & $57(4)$ & 3.7 & $60(7)$ & 4.3 \\
\hline
\end{tabular}

the fine-structure splittings of the metastable state are precisely known [31-35, 44], the fine structure of the Rydberg states can be reconstructed in a straightforward manner, as illustrated in Fig. 4. The diamonds in Fig. 4a) give the relative positions of the three lines observed experimentally (see examples in Fig. 2). The dashed lines correspond to the fine-structure intervals in the metastable state, and the differences indicated by the shaded regions represent the fine structure of the Rydberg states, which are listed in Table II. This structure is compared in Fig. 4b) with the fine structure derived from the eigenvalues of Eq. (4) after optimizing the values of $\gamma^{+}$(best value $-5.14(9) \mathrm{MHz}$ ) and $\gamma_{\mathrm{D}}^{+}$(best value $1.30(17) \mathrm{kHz}$ ) in a nonlinear least-squares fit (solid lines). The fine-structure intervals calculated with these constants are also compared to the experimentally determined intervals in Table II. The deviations only exceed $5 \mathrm{MHz}$ in four cases. The reduced- $\chi_{\nu}^{2}$ value of the fit is 1.05 indicating that our model describes the experimental data well. We also performed a MQDT analysis of the Rydberg spectrum using the methodology and program developed by Jungen and coworkers [37] and including the spins following the procedure described for
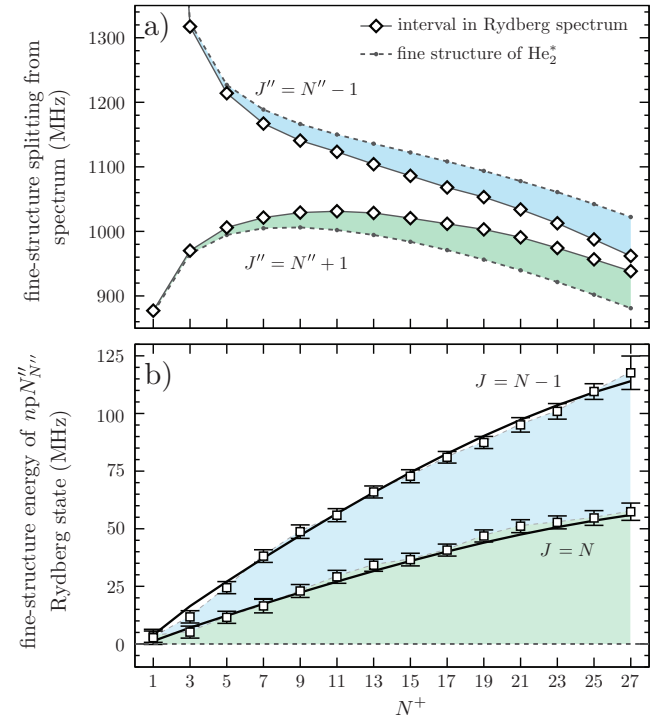

Figure 4 a) Fine-structure components, relative to the $J^{\prime \prime}=N^{\prime \prime}$ component, of Rydberg spectra recorded from $\mathrm{He}_{2}^{*}$, as a function of $N^{+}$(diamonds) and fine-structure intervals of $\mathrm{He}_{2}^{*}$ (dashed lines) [31-35, 44]. The green and blue shaded regions indicate the spin-rotation fine structure of the Rydberg states. b) Extracted fine-structure of Rydberg states relative to the $J^{\prime \prime}=N^{\prime \prime}+1$ component (squares) compared to model calculations (solid lines).

Table III. Predicted frequencies of magnetic-dipole transitions of $\mathrm{He}_{2}{ }^{+}\left(X^{+2} \Sigma_{\mathrm{u}}^{+}, v^{+}=0\right)$.

\begin{tabular}{cc}
\hline \hline$N^{+}\left(J^{+^{\prime}}\right) \leftarrow N^{+}\left({J^{+}}^{\prime \prime}\right)$ & Frequency $(\mathrm{MHz})$ \\
\hline $1(1 / 2) \leftarrow 1(3 / 2)$ & $7.69(14)$ \\
$3(5 / 2) \leftarrow 3(7 / 2)$ & $17.91(32)$ \\
$5(9 / 2) \leftarrow 5(11 / 2)$ & $28.0(6)$ \\
\hline \hline
\end{tabular}

$\mathrm{H}_{2}$ in Refs.[26, 39], but with the quantum-defect parameters obtained for the triplet states of $\mathrm{He}_{2}$ in Ref. 21. The analysis included the $\ell$-mixing interaction between $\mathrm{p}$ and $\mathrm{f}$ triplet Rydberg states of $\mathrm{He}_{2}$, first observed at $n=4$ in Ref. 45. Such interactions scale as $n^{-3}$ and lead to level shifts that are much less than the widths of the lines observed in our spectra at $n=50$, and tend to affect all fine-structure components in the same way. This analysis led to $\gamma^{+}$and $\gamma_{D}^{+}$values of $-5.04(9) \mathrm{MHz}$ and $1.22(17) \mathrm{kHz}$, respectively, in perfect agreement with the results obtained with Eq. (4). With $\gamma^{+}$and $\gamma_{D}^{+}$, the frequencies of the magnetic-dipole transitions connecting the fine-structure levels of the lowest rotational states of $\mathrm{He}_{2}{ }^{+}$that are expected to be the most relevant for astrophysics, are predicted with an accuracy of better than $1 \mathrm{MHz}$ (see Table III).

We have demonstrated a new method to precisely measure fine-structure intervals in molecular ions and showed how they are related to the fine structure of the corresponding Rydberg states. Applying this method to $\mathrm{He}_{2}{ }^{+}$, we determined the spin-rotation fine structure of 
the ground vibronic state for the first time. The spinrotation coupling constant significantly differs from the value derived by scaling the value calculated $a b$ initio for ${ }^{3} \mathrm{He}^{4} \mathrm{He}^{+}[18]$ (see above). When combined with the rotational term values of the $\mathrm{X}^{+2} \Sigma_{\mathrm{u}}^{+}\left(v^{+}=0\right)$ state of $\mathrm{He}_{2}{ }^{+}$reported in Ref. 23, the present data provides the complete level structure of the vibronic ground state of $\mathrm{He}_{2}{ }^{+}$.

We thank H. Schmutz and J. A. Agner for technical assistance and M. Beyer for useful discussions. This work was supported by the Swiss National Science Foundation (Grant No. 200020-172620) and by the European Research Council (Horizon 2020, Advanced Grant 743121).

*merkt@xuv.phys.chem.ethz.ch

[1] E. Majorana, Nuovo Cimento 8, 22 (1931).

[2] L. Pauling, J. Chem. Phys. 1, 56 (1933).

[3] J. Xie, B. Poirier, and G. I. Gellene, J. Chem. Phys. 122, 184310 (2005).

[4] W.-C. Tung, M. Pavanello, and L. Adamowicz, J. Chem. Phys. 136, 104309 (2012).

[5] C. B. Collins and W. W. Robertson, J. Chem. Phys. 40, 2208 (1964).

[6] B. E. Callicoatt, K. Förde, L. F. Jung, T. Ruchti, and K. C. Janda, J. Chem. Phys. 109, 10195 (1998).

[7] S. Lepp, P. C. Stancil, and A. Dalgarno, J. Phys. B: At. Mol. Opt. Phys. 35, R57 (2002).

[8] V. I. Korobov, L. Hilico, and J.-P. Karr, Phys. Rev. A 89, 032511 (2014).

[9] K. Piszczatowski, G. Łach, M. Przybytek, J. Komasa, K. Pachucki, and B. Jeziorski, J. Chem. Theory Comput. 5, 3039 (2009).

[10] M. Puchalski, J. Komasa, P. Czachorowski, and K. Pachucki, Phys. Rev. Lett. 117, 263002 (2016).

[11] T. Steimle and W. Ubachs, J. Mol. Spectrosc. 300, 1 (2014).

[12] W. Ubachs, J. C. J. Koelemeij, K. S. E. Eikema, and E. J. Salumbides, J. Mol. Spectrosc. 320, 1 (2016).

[13] E. J. Salumbides, J. C. J. Koelemeij, J. Komasa, K. Pachucki, K. S. E. Eikema, and W. Ubachs, Phys. Rev. D 87, 112008 (2013).

[14] E. J. Salumbides, A. N. Schellekens, B. Gato-Rivera, and W. Ubachs, New J. Phys. 17, 033015 (2015).

[15] A. Antognini, F. Nez, K. Schuhmann, F. D. Amaro, F. Biraben, J. M. R. Cardoso, D. S. Covita, A. Dax, S. Dhawan, M. Diepold, L. M. P. Fernandes, A. Giesen, A. L. Gouvea, T. Graf, T. W. Hänsch, P. Indelicato, L. Julien, C.-Y. Kao, P. Knowles, F. Kottmann, E.O. L. Bigot, Y.-W. Liu, J. A. M. Lopes, L. Ludhova, C. M. B. Monteiro, F. Mulhauser, T. Nebel, P. Rabinowitz, J. M. F. dos Santos, L. A. Schaller, C. Schwob, D. Taqqu, J. F. C. A. Veloso, J. Vogelsang, and R. Pohl, Science 339, 417 (2013).

[16] R. Pohl, F. Nez, L. M. P. Fernandes, F. D. Amaro, F. Biraben, J. M. R. Cardoso, D. S. Covita, A. Dax, S. Dhawan, M. Diepold, A. Giesen, A. L. Gouvea, T. Graf, T. W. Hänsch, P. Indelicato, L. Julien, P. Knowles, F. Kottmann, E.-O. L. Bigot, Y.-W. Liu,
J. A. M. Lopes, L. Ludhova, C. M. B. Monteiro, F. Mulhauser, T. Nebel, P. Rabinowitz, J. M. F. dos Santos, L. A. Schaller, K. Schuhmann, C. Schwob, D. Taqqu, J. F. C. A. Veloso, A. Antognini, and T. C. Collaboration, Science 353, 669 (2016).

[17] N. Yu and W. H. Wing, Phys. Rev. Lett. 59, 2055 (1987).

[18] N. Yu, W. H. Wing, and L. Adamowicz, Phys. Rev. Lett. 62, 253 (1989).

[19] A. Carrington, C. H. Pyne, and P. J. Knowles, J. Chem. Phys. 102, 5979 (1995).

[20] M. Raunhardt, M. Schäfer, N. Vanhaecke, and F. Merkt, J. Chem. Phys. 128, 164310 (2008).

[21] D. Sprecher, J. Liu, T. Krähenmann, M. Schäfer, and F. Merkt, J. Chem. Phys. 140, 064304 (2014).

[22] P. Jansen, L. Semeria, L. E. Hofer, S. Scheidegger, J. A. Agner, H. Schmutz, and F. Merkt, Phys. Rev. Lett. 115, 133202 (2015).

[23] L. Semeria, P. Jansen, and F. Merkt, J. Chem. Phys. 145, 204301 (2016).

[24] K. B. Jefferts, Phys. Rev. Lett. 23, 1476 (1969).

[25] Z. W. Fu, E. A. Hessels, and S. R. Lundeen, Phys. Rev. A 46, R5313 (1992).

[26] A. Osterwalder, A. Wüest, F. Merkt, and C. Jungen, J. Chem. Phys. 121, 11810 (2004).

[27] J. M. Brown and A. Carrington, Rotational Spectroscopy of Diatomic Molecules (Cambridge University Press, Cambridge, 2003).

[28] H. Lefebvre-Brion and R. W. Field, The Spectra and Dynamics of Diatomic Molecules (Academic Press, San Diego, 2004).

[29] M. Motsch, P. Jansen, J. A. Agner, H. Schmutz, and F. Merkt, Phys. Rev. A 89, 043420 (2014).

[30] P. Jansen, L. Semeria, and F. Merkt, J. Mol. Spectrosc. 322, 9 (2016).

[31] W. Lichten, M. V. McCusker, and T. L. Vierima, J. Chem. Phys. 61, 2200 (1974).

[32] W. Lichten and T. Wik, J. Chem. Phys. 69, 98 (1978).

[33] M. Kristensen and N. Bjerre, J. Chem. Phys. 93, 983 (1990).

[34] I. Hazell, A. Norregaard, and N. Bjerre, J. Mol. Spectrosc. 172, 135 (1995).

[35] C. Focsa, P. F. Bernath, and R. Colin, J. Mol. Spectrosc. 191, 209 (1998).

[36] U. Fano, Phys. Rev. A 2, 353 (1970).

[37] C. Jungen, in Handbook of High-Resolution Spectroscopy, "Elements of Quantum Defect Theory", Vol. 1 (John Wiley \& Sons, 2011).

[38] D. R. Yarkony, J. Chem. Phys. 90, 7164 (1989).

[39] C. Haase, M. Beyer, C. Jungen, and F. Merkt, J. Chem. Phys. 142, 064310 (2015).

[40] C. Jungen, and G. Raseev, Phys. Rev. A 57, 2407 (1998).

[41] R. N. Zare, Angular Momentum: Understanding Spatial Aspects in Chemistry and Physics (John Wiley \& Sons, New York, 1988).

[42] S. Fredin, D. Gauyacq, M. Horani, C. Jungen, G. Lefevre, and F. Masnou-Seeuws, Mol. Phys. 60, 825 (1987).

[43] R. W. Field, C. M. Gittins, N. A. Harris, and C. Jungen, J. Chem. Phys. 122, 184314 (2005).

[44] L. Semeria, P. Jansen, J. A. Agner, H. Schmutz, and F. Merkt, "Unpublished results," (2017).

[45] G. Herzberg and C. Jungen, J. Chem. Phys. 84, 1181 (1986). 